\title{
Cuanto más publicamos, menos nos citan
}

\author{
Jaime R. Rau'; Adrian Monjeau²; J. Cristobal Pizarro ${ }^{3, \bowtie}$ \& Christopher B. \\ ANDERSON ${ }^{4,5}$
}

\begin{abstract}
${ }^{1}$ Laboratorio de Ecología, Departamento de Ciencias Biológicas y Biodiversidad, Universidad de Los Lagos, Campus Osorno. Osorno, Chile. ${ }^{2}$ Departamento de Análisis de Sistemas Complejos de Fundación Bariloche y CONICET. Bariloche, Argentina. ${ }^{3}$ Departamento de Manejo de Bosque y Medioambiente. Facultad de Ciencias Forestales. Universidad de Concepción. Concepción, Chile. ${ }^{4}$ Centro Austral de Investigaciones Científicas (CADIC-CONICET). Ushuaia, Tierra del Fuego, Argentina. ${ }^{5}$ Instituto de Ciencias Polares, Ambiente y Recursos Naturales, Universidad Nacional de Tierra del Fuego (ICPA-UNTDF). Ushuaia, Tierra del Fuego, Argentina.
\end{abstract}

\begin{abstract}
RESUMEN. Existe una preocupación acerca de que la presión que ejercen las instituciones académicas por aumentar el número de publicaciones en revistas de factor de impacto elevado podría ir en desmedro de la calidad y, en consecuencia, de la incidencia que los trabajos puedan tener en la comunidad científica internacional. Para poner a prueba esta hipótesis, en este trabajo realizamos un análisis epistemométrico y estadístico de los trabajos publicados entre 1975 y 2015 por ecólogos que trabajan en instituciones argentinas, brasileras, chilenas y mexicanas. Se registraron los valores para el índice-h (que mide la cantidad de artículos publicados y sus citas) como indicador de calidad científica, y el total de ítems publicados en las revistas que ocuparon los 10 primeros lugares de cada país como indicador de productividad científica. Si bien el número de publicaciones creció de forma exponencial en estos cuatro países, desde el 2000 se observa que el número de citaciones por artículo disminuyó marcadamente. Es decir, fomentar la productividad científica mediante el incentivo de aumentar el número de publicaciones en revistas de alto factor de impacto, la política científica reinante en los países latinoamericanos, no refleja necesariamente una mayor inserción de la ecología latinoamericana en la comunidad científica internacional, sino más bien que las actuales políticas científicas de estos países produjeron lo contrario. Publicar menos trabajos, pero de mayor calidad y profundidad, o bien dedicarnos a fortalecer un sistema científico y tecnológico que se vincule a las demandas locales y regionales (y evaluar los productos acorde a esta meta) parecen ser caminos alternativos, pero más sabios, para construir una ecología regional con más impacto, relevancia, pertenencia y visibilidad globales.
\end{abstract}

[Palabras clave: índice-h, factor de impacto, productividad científica, ecología, América Latina]

\begin{abstract}
Aвstract. The more we publish, the less they cite us. There is a concern that the pressure to increase the number of papers in high impact factor journals could be detrimental to the quality of research, and therefore, to the impact that it might have in the international scientific community. In this work, we have done a scientometric and statistical analysis of published articles by Argentine, Brazilian, Chilean, and Mexican ecologists from 1975 to 2015, to test this hypothesis. H-index values (which measures an article's number of citations and total items published) were recorded in the top 10 journals for each country. While the number of publications grew exponentially in these four countries since 2000, we observed that the number of citations decreased markedly, which is to say that promoting scientific productivity by stimulating an increase in the number of publications in high impact factor journals, the dominant scientific policy in many Latin American countries, does not necessarily reflect greater insertion into the international scientific debate, but rather the current scientific policies have produced the opposite outcome. Publishing fewer papers, but with greater quality and depth, or perhaps dedicating ourselves to strengthening scientific and technological systems that are linked with local and regional needs (and evaluated accordingly), could be an alternative, but wiser, path to build a regional ecology with greater global impact, relevance, pertinence and visibility.
\end{abstract}

[Palabras clave: h-index, impact factor, research productivity, ecology, Latin America]

\section{INTRODUCCIÓN}

En un artículo reciente, Monjeau et al. (2015) realizaron un análisis general sobre un problema profundo: la evaluación del desempeño científico en América Latina y su relación con las políticas de incentivos y castigos que implementaron en nuestros países en los últimos años. El preconcepto que cuestionaremos aquí es la hipotética vinculación, fomentada por las políticas científicas en muchos países de Latinoamérica,

Editor asociado: Esteban Jobbágy entre la cantidad de trabajos científicos publicados en revistas indizadas (y de supuesto "alto factor de impacto") y su calidad como contribución científica.

Esta hipótesis es fácilmente falsada en muchos ámbitos no científicos. Por ejemplo, la escasa o nula vinculación entre calidad y cantidad es obvia en la música, la literatura y en las artes en general; grandes músicos, escritores y artistas no deben su "inmortalidad" a la cantidad de obras producidas, sino a las grandes (a veces 
pocas) obras que tuvieron incidencia en la cultura universal. En la ciencia, si bien los grandes nombres también siguen este patrón (e.g., nadie recuerda a Darwin por haber publicado cientos de "papers"), el sistema de evaluación que hemos diseñado en las últimas décadas se ha basado, en gran parte, en el reconocimiento de la calidad del trabajo de un científico según la cantidad y el factor de impacto (FI) de sus artículos publicados. Se ha llegado a un absurdo tan extremo que es como si en el arte se juzgara únicamente el aporte de un pintor por la cantidad de cuadros que pintó y que presentó en un selecto grupo de galerías, y no en cómo el trabajo y las obras de este pintor inspiraron a otros artistas y contribuyeron a la historia y el desarrollo de las artes visuales, como campo de saber y expresión humana.

Como forma de medir la calidad, los sistemas de evaluación científico-tecnológico globales adoptaron como indicador el FI de ThompsonReuters, actualmente Clarivinder Analytics. En publicaciones anteriores argumentamos, junto a un número creciente de autores, que esta estrategia es una manera errónea de evaluar las trayectorias individuales (Monjeau et al. 2013; Anderson et al. 2015a; Monjeau et al. 2015; Bohannon 2016), y que el mero énfasis en publicar la mayor cantidad de trabajos posibles únicamente en la colección de revistas "ISI" a la larga puede ser contraproducente tanto para la calidad de los trabajos como para el ambiente laboral que precisa una "buena" ciencia (Fischer et al. 2012; Sarewitz 2016). A pesar de esta crítica, los sistemas científico-tecnológicos de nuestros países en Latinoamérica realizaron esfuerzos multimillonarios para pertenecer a la circulación global del conocimiento, una estrategia que hasta hoy ha mostrado recompensas limitadas. Por ejemplo, a pesar de un crecimiento exponencial de las publicaciones en ecología desde 1975, la contribución de América Latina al mundo ISI no llegó al 4\% en 2015, y la mayoría de nuestras contribuciones se concentra en los cuartiles más bajos (Q3 y Q4) del ranking de los FI (ver ejemplo de Chile en SCImago 2015). Aun así, la evaluación curricular del Fondo Nacional de Desarrollo Científico y Tecnológico (FONDECYT) en Chile para proyectos de investigación, iniciación y postdocs en el campo Biología I (que incluye la ecología y las ciencias ambientales) es únicamente la sumatoria de sus publicaciones ISI multiplicadas por el FI del postulante y su patrocinante, cuando corresponde.
Otro indicador para medir la incidencia de un trabajo en la comunidad científica (léase ISI-angloparlante), es el índice-h, que mide con igual ponderación la cantidad de artículos publicados y también las veces que cada trabajo ha sido citado por otros. Este indicador robusto será siempre una cantidad menor que el número de artículos en los que se basa (Braun et al. 2005). Podrá aumentar de forma lineal con el tiempo, pero no disminuir, y será igual a cero cuando los artículos no tengan citas (Bornmann and Daniel 2007; Rau 2007). Puede razonarse que si un trabajo resulta muy citado es porque fue visible y útil a otros para desarrollar ciencia y, de alguna manera indirecta, el índice-h sería un mejor indicador de calidad (que cantidad y FI), y la epistemometría es la ciencia que analiza la producción de artículos científicos y sus citas a través de indicadores de calidad y visibilidad (Krauskopff 1994). Si al mundo globalizado le interesan nuestros desarrollos, el índice-h, como indicador epistemométrico, debería aumentar al ritmo de la cantidad de publicaciones científicas que insertamos en el sistema y sería un reconocimiento legítimo a la calidad de nuestras ideas. Por ello, en esta nueva contribución queremos examinar esta relación entre la cantidad y la evolución del índice-h de los trabajos publicados en revistas internacionales de ecología por científicos en Argentina, Brasil, Chile y México, desde 1975 hasta el 2015. Decidimos también investigar dónde estamos publicando y ofrecer un análisis crítico de estas tendencias que apuntan a evaluar el desarrollo de la ecología latinoamericana y su impacto mundial.

\section{Materiales y Métodos}

\section{Análisis epistemométrico}

Se obtuvieron las fuentes de documentos publicados por ecólogos que trabajan en instituciones de México, Brasil, Argentina y Chile desde la Web of Science ${ }^{T M}$ Core Collection (WoS) para 8 quinquenios que abarcaron el lapso 1975-2015. En junio de 2016 se buscó por categorías y esta información se refinó para la subcategoría Ecología. De esta manera se registraron para cada uno de esos países ("Address") los valores para el índice-h, citas por artículo y el total de ítems publicados para cada quinquenio. A fin de obtener valores discretos del índice-h para las ventanas de tiempo ("Timespan") consideradas se utilizó el comando "Times Cited" que entrega los valores del índice para cada quinquenio utilizado (Nathan Sutton, comunicación 
personal). Si bien es sabido que los mayores índices de citas anuales corresponden a los artículos más recientes (Jaksic 1994), debe considerarse que ese comando calcula el índice-h para el período de tiempo indicado por el usuario (en nuestro caso, quinquenios), por lo que las citas no se acumulan entre los diferentes quinquenios comparados $\mathrm{y}$, además, porque tampoco lo hacen los artículos ya que son todos distintos entre quinquenios (ver http://images.webofknowledge.com/ WOKRS523_2R2/help/WOS/hp_citation_ report_hindex.html).

En efecto, el algoritmo para el comando "Times Cited", usado para calcular el índiceh, tiene solo dos etapas: 1) se ordenan por rangos las citas que reciben los artículos de un autor, y 2) se contabiliza desde el artículo más citado hasta que el recuento no sea mayor que el número total de citas del siguiente artículo (cf. Rau 2007). Para cada país, además, se identificaron los nombres de las revistas que ocuparon los 10 primeros lugares respecto de sus citas acumuladas para el quinquenio 2010-2015. Para estas revistas se obtuvieron sus FI para el período 2014-2015 desde el portal ResearchGate, y sus cuartiles (Q1 más impacto y Q4 menos impacto) del WoS que usa la edición 2015 del Journal Citation Reports.

\section{Análisis estadístico}

Se utilizó el paquete computacional en línea VassarStats (http://www.vassarstats.net) para obtener la media aritmética y su error estándar. Se usó el paquete computacional Stat en lenguaje R(RDevelopment Core Team 2015) para ajustar los datos del número total de ítems por quinquenio a una distribución exponencial con la prueba de Kolmogorov-Smirnov para una muestra (DN) y también para comparar los valores de tendencia central del índice-h y de los FI de las revistas que ocuparon los primeros 10 lugares de los países considerados mediante un análisis de varianza de una vía (F). El valor de la tasa anual de crecimiento se obtuvo con el empleo de fórmulas estándar descritas en Rau (1997).

\section{Resultados}

La producción de artículos indizados en el WoS por los ecólogos chilenos, mexicanos y brasileros comenzó en el quinquenio 19751979, y un quinquenio más tarde para el caso de los ecólogos argentinos (1980-1984) (Tabla 1). En Argentina y Chile, durante el quinquenio 1995-1999, aun cuando el número de ítems publicados creció de forma exponencial (47\% en Chile y 55.4\% en Argentina) (DN=0.106, $P=0.999$ y $\mathrm{DN}=0.145$,

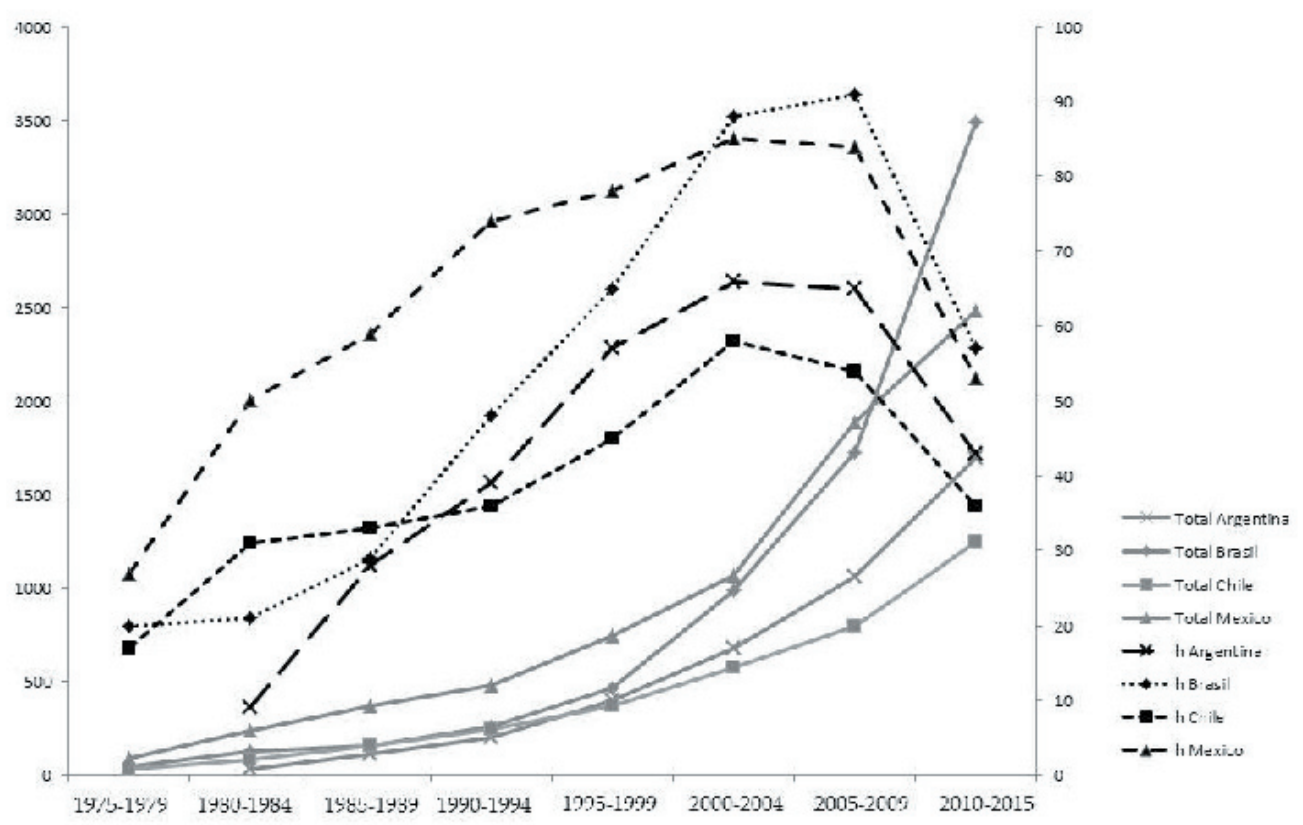

Figura 1. Tendencias en el número de publicaciones (líneas sólidas) versus sus índices-h para artículos del área de la ecología publicados en Argentina (cruces), Brasil (rombos), Chile (cuadrados) y México (triángulos). Datos obtenidos del análisis de la Web of Science en intervalos de 5 años.

Figure 1. Trends in the number of publications (solid lines) versus their h-index (dashed lines) for ecology-related articles in Argentina (crosses), Brazil (diamonds), Chile (squares) and México (triangles). Data obtained from an analysis the Web of Science database in 5-year intervals. 
Tabla 1. Indicadores epistemométricos (WoS) para la disciplina ecológica del período 1975-2015 en Chile (A), Argentina (B), México (C) y Brasil (D).

Table 1. Scientometric indicators (WoS) for the discipline of ecology for the period 1975-2015 in Chile (A), Argentina (B), México (C) and Brazil (D).

\begin{tabular}{|c|c|c|c|c|c|c|}
\hline & A) CHILE & & & B) ARGENTIN & & \\
\hline Quinquenio & $\mathrm{H}$ & Citas/artículo & Total ítems & $\mathrm{H}$ & Citas/artículo & Total ítems \\
\hline 1975-1979 & 17 & 32.0 & 29 & -- & -- & -- \\
\hline 1980-1984 & 31 & 32.5 & 80 & 9 & 8.5 & 35 \\
\hline 1985-1989 & 33 & 22.7 & 154 & 28 & 22.1 & 114 \\
\hline 1990-1994 & 36 & 17.6 & 248 & 39 & 27.2 & 199 \\
\hline 1995-1999 & 45 & 24.7 & 369 & 57 & 35.6 & 402 \\
\hline $2000-2004$ & 58 & 25.8 & 575 & 66 & 30.2 & 680 \\
\hline 2005-2009 & 54 & 20.8 & 796 & 65 & 24.3 & 1062 \\
\hline $2010-2015$ & 36 & 6.9 & 1246 & 43 & 7.6 & 1695 \\
\hline \multirow{2}{*}{$\begin{array}{l}\text { Mediaterror } \\
\text { estándar }\end{array}$} & $38.7 \pm 4.7$ & $22.9 \pm 2.9$ & $437.12 \pm 147.40$ & $43.85 \pm 7.86$ & $22.2 \pm 4.0$ & $598.1 \pm 227.5$ \\
\hline & C) MEXICO & & & D) BRASIL & & \\
\hline Quinquenio & $\mathrm{H}$ & Citas/artículo & Total ítems & $\mathrm{H}$ & Citas/artículo & Total ítems \\
\hline 1975-1979 & 27 & 27.1 & 91 & 20 & 24.1 & 48 \\
\hline 1980-1984 & 50 & 40.0 & 240 & 21 & 11.7 & 128 \\
\hline 1985-1989 & 59 & 62.0 & 372 & 29 & 19.6 & 154 \\
\hline 1990-1994 & 74 & 39.5 & 480 & 48 & 26.6 & 261 \\
\hline 1995-1999 & 78 & 36.1 & 742 & 65 & 33.0 & 469 \\
\hline $2000-2004$ & 85 & 34.7 & 1069 & 88 & 34.7 & 987 \\
\hline 2005-2009 & 84 & 24.4 & 1883 & 91 & 30.3 & 1727 \\
\hline $2010-2015$ & 53 & 8.3 & 2484 & 57 & 7.8 & 3489 \\
\hline $\begin{array}{l}\text { Media } \pm \text { error } \\
\text { estándar }\end{array}$ & $63.7 \pm 7.1$ & $34.0 \pm 5.4$ & $920.1 \pm 300.9$ & $52.4 \pm 9.9$ & $23.5 \pm 3.5$ & $907.9 \pm 419.7$ \\
\hline
\end{tabular}

Tabla 2. Nómina de las 10 revistas de ecología con los FI más altos para Chile (A), Argentina (B), México (C) y Brasil (D).

Table 2. Names of the 10 ecology journal with the highest Impact Factor (IF) for Chile (A), Argentina (B), Mexico (C) and Brazil (D).

\begin{tabular}{|c|c|c|c|}
\hline \multicolumn{2}{|l|}{ A) CHILE } & \multicolumn{2}{|l|}{ B) ARGENTINA } \\
\hline Revista & $\begin{array}{c}\text { FI } \\
2014 / 2015 \\
\end{array}$ & Revista & $\begin{array}{c}\text { FI } \\
2014 / 2015\end{array}$ \\
\hline (01) Global Change Biology & 8.04 & (01) Global Change Biology & 8.04 \\
\hline & 3.76 & (02) Ecology Letters & 10.68 \\
\hline & 10.68 & (03) Ecology & 4.65 \\
\hline $\begin{array}{l}\text { (03) Ecology Letters } \\
\text { (04) BioGeoSciences }\end{array}$ & 3.97 & (04) Biodiversity and Conservation & 2.36 \\
\hline $\begin{array}{l}\text { (05) Annual Review of Ecology, Evolution and } \\
\text { Systematics }\end{array}$ & 10.56 & (05) Biological Conservation & 3.76 \\
\hline $\begin{array}{l}\text { (06) ISME Journal } \\
\text { (07) Ecography }\end{array}$ & 9.30 & (06) Ecology Letters & 10.68 \\
\hline \multirow{2}{*}{$\begin{array}{l}\text { (07) Ecography } \\
(08) \text { Ecology Letters }\end{array}$} & 4.77 & (07) Molecular Ecology & 6.49 \\
\hline & 10.68 & (08) Ecology Letters & 10.68 \\
\hline (09) Diversity and Distributions & 3.66 & (09)Trends in Ecology and Evolution & 16.19 \\
\hline \multirow[t]{2}{*}{ (10) Ecology Letters } & 10.68 & (10) Austral Ecology & \\
\hline & $7.65 \pm 1.0$ & Media $\pm \mathrm{e}$ & $7.56 \pm 1.44$ \\
\hline \multicolumn{2}{|l|}{ C) MEXICO } & \multicolumn{2}{|l|}{ D) BRASIL } \\
\hline \multirow{2}{*}{$\begin{array}{l}\text { (01) Evolution } \\
\text { (02) Ecography }\end{array}$} & 4.68 & (01) Ecography & 4.77 \\
\hline & 4.77 & $\begin{array}{l}\text { (02) Bulletin of the American Museum of } \\
\text { Natural History }\end{array}$ & 4.54 \\
\hline $\begin{array}{l}\text { (03) Ecology } \\
\text { (04) Journal of Wildlife Management }\end{array}$ & 4.65 & (03) Biological Conservation & 3.76 \\
\hline & 1.94 & (04) Oecologia & 2.50 \\
\hline & 10.86 & (05) ISME Journal & 9.30 \\
\hline $\begin{array}{l}(05) \text { Ecology Letters } \\
(06) \text { Ecology }\end{array}$ & 4.65 & (06)Conservation Biology & 2.70 \\
\hline & 4.84 & (07) Global Ecology and Biogeography & 6.76 \\
\hline (08) Ecological Applications & 4.55 & (08) Ecological Applications & 4.55 \\
\hline & 3.91 & (09) Global Ecology and Biogeography & 6.76 \\
\hline \multirow{2}{*}{\multicolumn{2}{|c|}{$\begin{array}{rc}\text { (10) Annual Review of Ecology and Systematics } & 9.55 \\
& \text { Media } \pm \text { error estándar } 5.44 \pm 0.84\end{array}$}} & (10) Ecological Economics & 4.17 \\
\hline & & Media \pm error estándar & $4.98 \pm 0.65$ \\
\hline
\end{tabular}


$P=0.998$, respectivamente) sus citas y el índice-h decrecieron $(\mathrm{DN}=0.106, P=0.999 \mathrm{y}$ $\mathrm{DN}=0.145 ; P=0.998$, respectivamente). Para Brasil y México se observaron tendencias similares, con 53.6\% (DN=0.250, $P=0.699)$ y $41.3 \%$ (DN=0.121, $P=0.999$, respectivamente) (Figura 1).

Los valores de tendencia central del índiceh para los cuatro países comparados no presentaron diferencias estadísticamente significativas $(F=2.09, P=0.125$, g.1.=3.27). En el caso de los FI de las revistas que ocuparon los 10 primeros lugares, tampoco se encontraron diferencias estadísticamente significativas $(F=1.81, P=0.164$, g.1.=3.36). En la Tabla 2 se presentan las 10 revistas de ecología por país que tuvieron los FI más altos. Todas las revistas donde publicaron ecólogos chilenos correspondieron al Q1, mientras que para Argentina fueron 9 de 10 (una revista en el Q3), lo mismo que para México (una revista en el Q3). Brasil presentó 8 de 10 revistas en el Q1 (dos revistas en el Q2).

\section{DisCUSIÓN}

Las reglas y convenciones sobre diversas cosas, incluyendo estándares de calidad, han suscitado el interés de filósofos e intelectuales. Por ejemplo, el filósofo de historia Charles Taylor, en su ensayo Siguiendo Una Regla (1997), relata cómo las reglas son, en realidad, prácticas culturales en las que sus seguidores actúan sin necesariamente articular cognitiva y conscientemente el trasfondo (social o cultural) que originó la regla. De la misma forma, aunque hemos mostrado la ausencia de relación entre cantidad de artículos e indicadores epistemométricos, la explicación de este fenómeno, y de por qué insistimos en seguir la regla "cantidad de publicaciones científicas" en Latinoamérica requiere, al menos, de dos pasos fundamentales: 1) una articulación de una explicación para comprender qué sentido tiene esta regla, y 2) poner esta explicación de nuevo en el espacio social para su evaluación o re-articulación a través de foros, congresos, artículos de nuestro campo de acción (en este caso, la ecología).

Mientras el número de trabajos se mantiene al alza, el quinquenio 2000-2005 representa para la tendencia declinante del índice-h de todos los países analizados un punto de umbral calidad-cantidad, fenómeno que requiere de mayor investigación. En ese período, el crecimiento se estanca y prosigue a él un rápido declive, y adopta una característica forma de U-invertida. Antes que nosotros, Meneghini et al. (2008) encontraron un patrón similar en relación a la escasa cantidad de citas respecto de la gran cantidad de artículos publicados por autores latinoamericanos, sin encontrar tampoco información que avale si esto se debe a una calidad inferior de nuestros trabajos o a una tendencia psico-social hacia la poca consideración internacional de estos trabajos. Este fenómeno de U-invertida fue detectado también en la relación entre crecimiento económico y calidad de vida, en probable relación a cambios macro-políticos y la adopción de políticas neoliberales (i.e., Threshold hypotesis, Max-Neef 1995:118). Más allá de estas hipótesis, si nuestros sistemas científico-tecnológicos fuesen capaces de detectar esta situación, la estrategia de fomento científico de los países debería cambiar y optar por incentivar más calidad que cantidad después de llegar a ese umbral. A menos que nuestros artículos ahora sean "bellas durmientes", que resurjan con los años venideros y que generen muchas citas (Van Rann 2004), es sabido que mientras más se publica, la probabilidad de recibir citas por artículo disminuye (ver Jaksic 1994; Leimu and Koricheva 2004; Hirsch 2005; Meneghini et al. 2008). De hecho, para los ecólogos más citados de Chile, el número de artículos ISI publicados, el número de aquellos que no fueron citados y el índice-h se asociaron positiva y significativamente (Rau 2007), y su índice-h se relacionó tanto con el número de citas y aún más con el número de artículos (Rau 2007). Por otro lado, se conoce una paradoja en relación al patrón de citas y el país de origen de los autores de artículos científicos. Por ejemplo, en las ciencias biomédicas, Estados Unidos es el mayor productor de "papers" y, al mismo tiempo, esos artículos son citados al menos cinco veces más por autores de instituciones nacionales que extranjeras, excluyendo autocitas (Pasterkamp et al. 2007). Por el contrario y al mismo tiempo, autores latinoamericanos que apuntan en publicar en revistas de alto FI generan listas con referencias de autores y revistas prominentes, y evitan citar a sus compatriotas y trabajos en revistas locales (Meneghini et al. 2008).

El espacio social o campo de trabajo está también cambiando, lo que suma otro factor importante. Por ejemplo, la llegada y el auge del Open Access promete modificar tanto la conducta de editoriales y científicos, así como la indización de varias revistas y 
bases de datos (McVeigh 2004; Butler 2013). Respecto a cómo enfrentamos esta situación, cabe destacar que hemos encontrado en otros campos como la música, la sociología, la economía, las matemáticas y la filosofía más herramientas para la reflexión, el análisis y la autocrítica que en nuestro propio campo de trabajo. Es reconocido también que la formación de los ecólogos es débil en filosofía de la ciencia (Graham and Dayton 2002), por lo que en la consolidación de nuestro campo en Latinoamérica desarrollamos pocas herramientas para pensar y rectificar nuestro propio quehacer en función de nuestras realidades y sus cambios. Por ejemplo, siguiendo la teoría social de Bordieu (1991), al centrar la atención sólo en la producción de artículosestamosfragmentandonuestrocampo de acción y, por ende, perdiendo relevancia, tanto para nosotros mismos como para el interés que la sociedad rescata de nuestro trabajo (aplicación, extensión, divulgación y educación). En este sentido estamos reduciendo nuestro "habitus" científico (Bordieu 1991) a la repetición de patrones que adecuan la acción a lo conveniente, adoptan un ritmo de acción frenético donde no se socializa ni se dialoga sobre nuestros propios problemas (se pueden ver excepciones a esta regla en distintas contribuciones de la sección Debates de esta revista en los últimos años: Farji-Brenner 2012; Anderson et al. 2015b; García Curilaf and Denegri 2016). En este sentido, podríamos comenzar por dejar de pensar la ecología como un campo aislado y ajeno a otras realidades del mundo y centrarnos en potenciar que los nuevos ecólogos se dispongan a pensar su propia ciencia y crear conceptos y teorías, más allá de refinar o adaptar marcos teóricos foráneos o adoptar indicadores (cantidad de "papers", FI) que sustituyen el bosque del conocimiento por el uso radicalizado de parámetros que ni siquiera comprendemos y, en consecuencia, utilizamos de manera errónea.

En nuestra opinión, tendríamos que decidir enfocar nuestros esfuerzos entre dos tipos de incidencia: 1) intentar que nos miren más desde el primer mundo (seguir subordinados a las veleidades de las editoriales y conseguir de alguna forma más citaciones para nuestros artículos, Grigston and Mudrak 2016), ó 2) focalizar esfuerzos científicos y tecnológicos en temas de relevancia social y ambiental con alta incidencia en la realidad regional (Holbrook 2012; Anderson et al. 2015a). Para potenciar estos dos tipos de incidencia, un gran aporte sería contar, por ejemplo, con una gran revista de ecología latinoamericana (e.g., "Annual Review of Latin American Ecology") que publique al año 15 ó 20 trabajos científicos de síntesis en inglés y en español (Di Bitetti and Ferreras 2016). Estos artículos no sólo serían muy citados, sino también resultarían de alta relevancia para la enseñanza de la ecología, pues estarían enfocados en nuestros ecosistemas y biodiversidad, en vez de seguir las modas o necesidades del mundo y ecosistemas de otros países angloparlantes. La elaboración de estos artículos debería premiar tanto el liderazgo de ecólogos consolidados como el trabajo y colaboración de ecólogos jóvenes, situación muy diferente al actual sistema de incentivo económico y premio a la publicación de primeros autores.

Concluimos que un sistema regional y, especialmente, colaborativo en el desarrollo de la ecología latinoamericana ayudaría también a descentralizar el poder y diversificar el abanico de temas y aspectos a investigar, que sean innovadores y además socialmente relevantes (Anderson et al. 2015a).

Agradecimientos. Los autores agradecen a Fabián M. Jaksic por la revisión de una versión temprana del manuscrito. JRR agradece a Soraya Sade por su asistencia en varias fases de este trabajo, a Jorge Hirsch por ayudarnos con la interpretación de su propio índice $h$ y a Nathan Sutton (Clarivinder Analytics, actual propietaria del WoS), por clarificarnos el cálculo del índice-h no acumulativo en períodos discretos de tiempo. AM agradece a Fundación Bariloche por su ambiente creativo y de libertad intelectual. JCP agradece su beca postdoctoral latinoamericana CONICET Res. $\mathrm{N}^{\mathrm{o}} 3503$.

\section{REFERENCIAS}

Anderson, C. B., A. Monjeau, and J. R. Rau. 2015a. Knowledge dialogue to attain global scientific excellence and broader social relevance. BioScience 65:709-717.

Anderson, C. B., J. C. Pizarro, R. Estevez, A. Sapoznikow, A. Pauchard, O. Barbosa, A. Moreira-Muñoz, and A. E. J. Valenzuela. 2015b. ¿Estamos avanzando hacía una socio-ecología? Reflexiones sobre la integración de las dimensiones "humanas" en la ecología en el sur de América. Ecología Austral 25:263-272.

Bohannon, J. 2016. Hate journal impact factors? New study gives you one more reason. Science Doi: 1126/science/ aag0643.

Bordieu, P. 1991. Language and Symbolic Power. Harvard University Press. Cambridge, MA, USA. 
Bornmann, L., and H-D. Daniel. 2005. Does the h-index for ranking of scientist really work? Scientometrics 65:391392.

Braun T., W. Glänzel, and A. Schubert. 2005. A Hirsch-type index for journals. The Scientist 19:8.

Di Bitetti, J., and A. Ferreras. 2017. Publish (in English) or perish: The effect on citation rate of using languages other than English in scientific publications. Ambio 46(1): 121-127.

Farji-Brenner, A. 2012. El valor de tener muchas citas. Ecología Austral 22:215-220.

Fischer, J., E. G. Ritchie, and J. Hanspach. 2012. Academia's obsession with quantity. Trends in Ecology and Evolution 27:473-474.

García Curilaf, C. I., and G. M. Denegri. 2016. Supuestos epistemológicos y ontológicos presentes en la historia de la ecología. Ecología Austral 26:221-228.

Graham, M. H., and P. K. Dayton. 2002. On the evolution of ecological ideas: Paradigms and scietific progress. Ecology 83:1481-1489.

Grigston, J., and B. Mudrak. 2016. The State of Authorship. Maximizing Impact with the Time and Money You Spend. American Journal Experts. Durham, N.C. Pp. 11.

Hirsch, J. E. 2005. An index to quantify individual's scientific research output. Proceedings of National Academy of Sciences USA 102:16569-16572.

Holbrook, J. B. 2012. Re-assessing the science-society relation: The case of the US National Science Foundation's broader impacts merit review criterion (1997-2011). Pp. 328-362 in R. Frodeman, J. B. Holbrook and H. Xiaonan (eds.). Peer Review, Research Integrity, and the Governance of Science - Practice, Theory, and Current Discussions. People's Publishing House. Beijing, China.

Jaksic, F. M. 1994. Artículos clásicos, modas e impacto en Ecología: los ecólogos chilenos en el contexto internacional, regional y local. Revista Chilena de Historia Natural 67:245-251.

Krauskopff, M. 1994. Epistemometria, a term contributing to express the meaning and potential methodologies of scientometrics in Spanish speaking countries. Scientometrics 30:425-428.

Leimu, R., and J. Koricheva. 2004. What determines the citation frequency of ecological papers? Trends in Ecology and Evolution 20:28.32.

Meneghini, R., A. L. Packer, and L. Nassi-Cal'. 2008. Articles by Latin American authors in prestigious journals have fewer citations. PLoS ONE 3(11):e3804.

Max-Neef, M. 1995. Economic growth and quality of life: a threshold hypothesis. Ecological Economics 15:115-118.

McVeigh. 2004. Open Access Journals in the ISI Citation Databases: Analysis of Impact Factors and Citation Patterns. A citation study from Thomson Scientific. Thomson Corporation. URL: http:/ /ip science.thomsonreuters.com/m/ pdfs/openaccesscitations2.pdf.

Monjeau, A., J. R. Rau, and C. B. Anderson. 2013. Regional science: Latin America should ditch Impact Factor. Nature 499:29.

Monjeau, A., J. R. Rau, and C. B. Anderson. 2015. El síndrome del factor de impacto y la ética ambiental en América Latina: ¿ha llegado el tiempo de la insurrección? Cuadernos de Ética. Edición especial: Ética Ambiental Latinoamericana: Contextos, Enfoques, Desafíos 30:1-23.

Pasterkamp, P., J. Rotmans, D. Kleijn, and C. Bors. 2007. Citation frequency: a biased measure of research impact significantly influenced by the geographical origin of research articles. Scientometrics 70:153-165.

R Development Core Team. 2015. R: A language and environment for statistical computing. R Foundation for Statistical Computing. Vienna, Austria.

Rau, J. R. 1997. Factores de impacto de la Revista Chilena de Historia Natural: 1991-1995. Revista Chilena de Historia Natural 70:453-457.

Rau, J. R. 2007. Índice h (2000-2004) de los científicos ambientales más citados que residen en Chile. Revista Chilena de Historia Natural 80:381-383.

Sarewitz, D. 2016. The pressure to publish pushes down quality. Nature 533:147.

SCImago. 2015. Principales indicadores cienciométricos de la actividad científica chilena. Informe 2015. Programa de Información Científica, CONICYT. Santiago, Chile.

Taylor, C. 1997. Seguir una regla. Pp. 221-238 in Argumentos filosóficos, ensayos sobre el conocimiento, el lenguaje y la modernidad. Paidós, Barcelona, España.

Van Rann, A. F. J. 2004. Sleeping beauties in science. Scientometrics 59(3):461-466 